\title{
Systematic Model Generation for Shear Stress using Elementary Mathematical Equation
}

\author{
Yatharth Joshi, Yuvraj Joshi, Gagan Bansal, Jasmeet Kalra
}

\begin{abstract}
In engineering mechanics we deal with the forces, tensions, deflections, stresses, etc. acting on a system in various directions. There are basically of two types of stresses i.e. normal stresses and shear stresses. Normal stresses further can be compressive or tensile. Also from these elementary stresses we derive other stresses such as bending, torsion, their combinations etc. In the current research model of shear stress we will develop that shear stresses are actually nothing but the combination of normal stresses acting on different directions and generations. We will look on various 'generations' of stresses acting in different directions due to shear forces. As through algebraic mathematics, any function can be represented by any polynomial equation which may be in the form of any finite or infinite series, similarly in this shear stress model we will further derive an equation in which shear stress can be represented as an infinite series of compressive and tensile stresses.
\end{abstract}

Keywords: Normal Stress, Direct Stress, Shear Stress, Strength of Material, Engineering Mechanics, Mechanical

\section{INTRODUCTION}

Basically we say that if the load is parallel to the crosssection of any member is shear force and that causes shear stress into the member [1]. Till now we used to study shear stress as shown below:

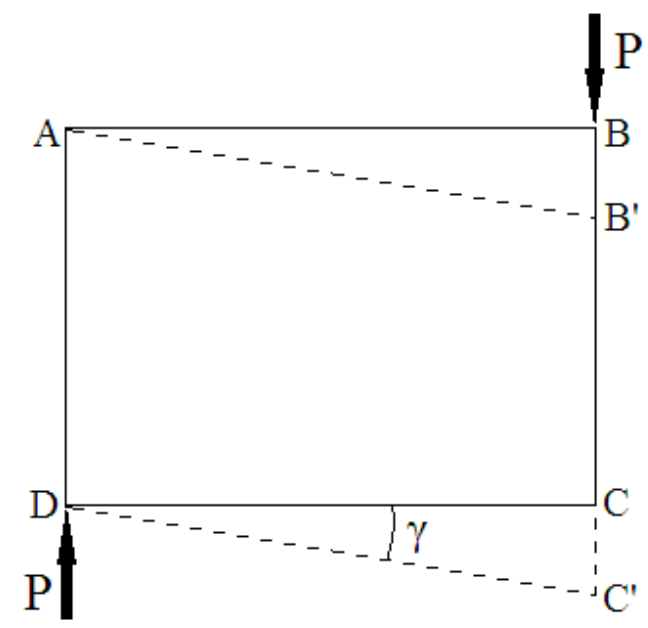

Figure 1: Representation of shear stress in classical model.

In this model shear stress,

$\tau=\gamma G$

Revised Manuscript Received on April 25, 2019.

*Corresponding Author

*Yatharth Joshi, M. Tech Scholar, Graphic Era deemed to be University, Dehradun, India. E-mail: yatharthjoshi2010@gmail.com

Yuvraj Joshi, M. Tech Scholar, Graphic Era deemed to be University, Dehradun, India.

Gagan Bansal, Assistant Professor, Department of Mechanical Engineering, Graphic Era deemed to be University, Dehradun, India.

Jasmeet Kalra, Assistant Professor, Department of Mechanical Engineering, Graphic Era Hill University, Dehradun, India.
Where,

$$
\begin{gathered}
\tau \leftrightarrow \text { shear stress } \\
\gamma \leftrightarrow \text { angle of deflection } \\
G \leftrightarrow \text { Bulk Modulus }
\end{gathered}
$$

Here $A B C D$ is a member upon which shear force $P$ is acting. $\mathrm{ABCD}$ is its initial position without any force acting and after $\mathrm{P}$ is acting, some deflection takes place and the position changes to $A B^{\prime} C^{\prime} D$.

On further investigating this classical model we found that there are some other stresses involved. Let us see following diagrams:

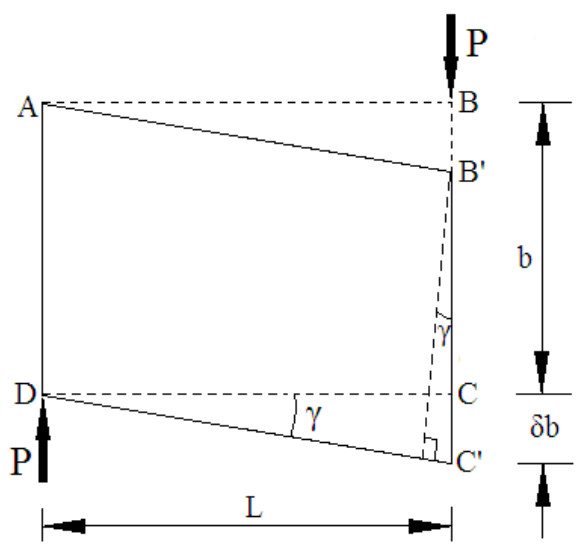

Figure 2a Dimensions of the elements under shear

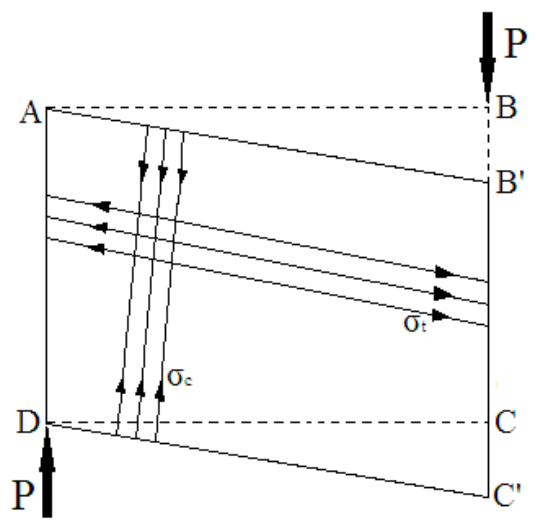

Figure 2b Stresses acting on the on the element under shear

From fig 2a,

$A B=D C=l$

$A D=B C=B^{s} C^{s}=b$

From geometry,

$$
A B^{\circ}=D C^{s}=\frac{\mathbb{1}}{\cos Y}
$$

And,

$B B^{s}=C C^{s}=\delta b$

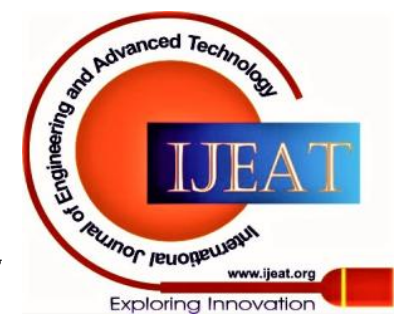




\section{Systematic Model Generation for Shear Stress using Elementary Mathematical Equation}

By fig 2b,

$\sigma_{t}=$ Tensile stress

\section{$\sigma_{c}=$ Compressive stress}

And $\sigma_{t}$ and $\sigma_{e}$ are perpendicular in direction.

The stresses $\sigma_{t}$ and $\sigma_{c}$ arises due to the tension and compression. If we look at $\mathrm{AB}^{\prime}$ and $\mathrm{DC}^{\prime}$ then, $\mathrm{AB} \mathrm{B}^{\prime}>\mathrm{AB}$ and $\mathrm{DC} '>\mathrm{DC}$ and the fibers of material of this member parallel to $\mathrm{AB}^{\prime}$ will experience pure tensile stress.

Similarly $\mathrm{B}$ ' $\mathrm{E}<\mathrm{B} \mathrm{C}^{\prime}$ or $\mathrm{B}{ }^{\prime} \mathrm{E}<\mathrm{BC}$, i.e. the fibers of material of this member parallel to $\mathrm{B}^{\prime} \mathrm{E}$ will experience pure compressive stress.

Now as we know that,

$\sigma \propto \epsilon$

i.e. stress is directly proportional to strain or,

$\sigma=E \varepsilon$

$\sigma_{\mathrm{t}}=E \epsilon_{\mathrm{t}} \quad$ or $\quad \sigma_{\mathrm{c}}=E \epsilon_{\mathrm{c}}$

Where,

$\epsilon_{\mathrm{t}}=$ Tensile strain

$\epsilon_{e}=$ Compressive strain

Now there is a big contradiction in my head that is Young's modulus is same or not for compression an tension. We know that there is term strength differential [2], which denotes the difference in the compressive about Young's modulus can be determined only by experiments.

Now,

$$
\begin{aligned}
& \epsilon_{\mathrm{t}}= \frac{A B^{s}-A B}{A B} \\
& \epsilon_{\mathrm{t}}= \frac{\frac{l}{\cos y}-l}{l} \\
& \epsilon_{\mathrm{t}}=\frac{1-\cos y}{\cos y} \quad \epsilon_{\mathrm{t}}=\frac{1}{\cos Y}-1
\end{aligned}
$$

And

$$
\begin{gathered}
\varepsilon_{c}=\frac{B^{\prime} C^{y}-B^{\prime} E}{B^{\prime} C^{r}}=\frac{B C-B^{\prime} E}{B C} \\
\varepsilon_{c}=\frac{b-b \cos \gamma}{b} \\
\epsilon_{c}=1-\cos \gamma
\end{gathered}
$$

Let there be the two cross-section areas, one perpendicular to $\sigma_{t}$ and one perpendicular to $\sigma_{e}$ as shown below:
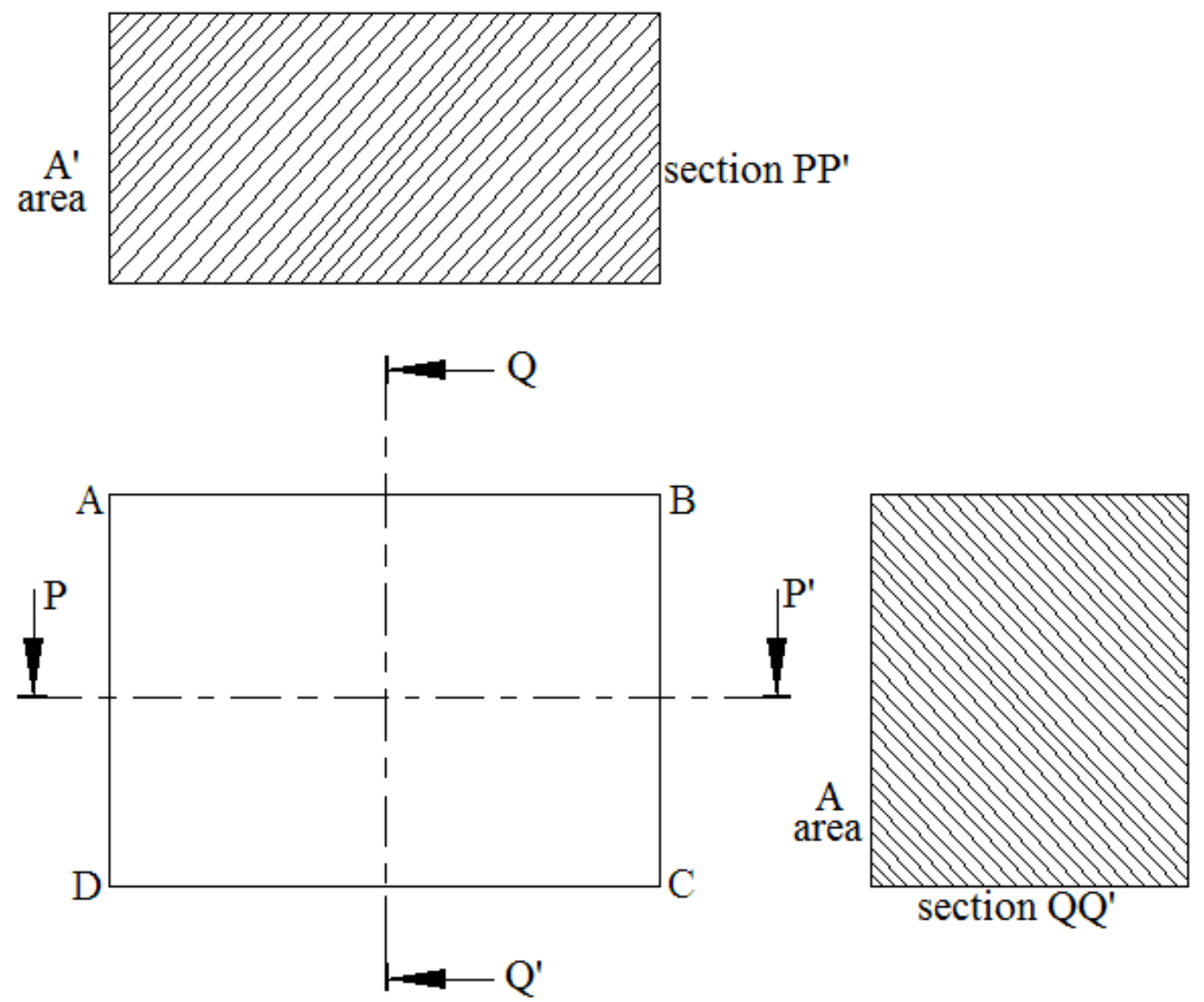

Figure 3: Representation of two different cross-sections of the member.

So, from equation $3 \& 4$ we can write:

$\sigma_{\mathrm{t}}=\left(\frac{1-\cos \gamma}{\cos Y}\right) E$

$\sigma_{c}=(1-\cos \gamma) E$
So the internal forces that arise due to these stresses are $F_{t}$ and $\mathrm{F}_{\mathrm{c}}$. 
Where,

$$
F_{\mathrm{t}}=\text { Internal force due to tension }
$$

$F_{c}=$ Internal force due to compression

But as shown in figure 3 and the direction of $\sigma_{t} \& \sigma_{c}$ in figure $2 \mathrm{~b}$, the area perpendicular to $\sigma_{\mathrm{t}}$ is $\mathrm{A}$ and area perpendicular to $\sigma_{c}$ is $\mathrm{A}^{\prime}$.

So $F_{t} \& F_{c}$ are:

$$
\begin{aligned}
& F_{\mathrm{t}}=\left(\frac{1-\cos \gamma}{\cos \gamma}\right) E A \\
& F_{c}=(1-\cos \gamma) E A^{\prime}
\end{aligned}
$$

This means that the internal forces $\mathrm{F}_{\mathrm{t}}$ and $\mathrm{F}_{\mathrm{c}}$ are compressive and tensile caused by the shear force $\mathrm{P}$. So it can be also said that the resultant of $F_{t}$ and $F_{c}$ is equal and opposite to the external shear force $\mathrm{P}$. This is due to the material of member would resist the external load.

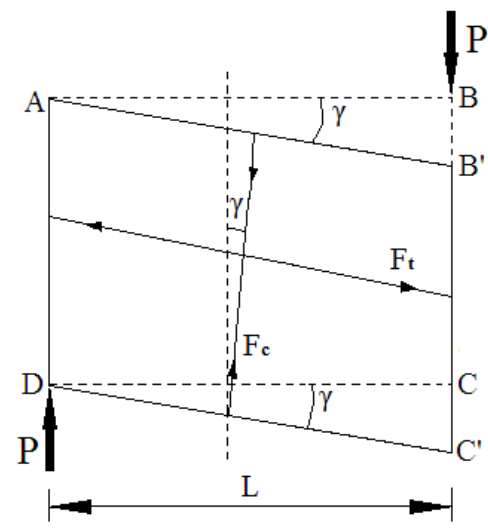

Figure 4a. Representation of $F_{t} \& F_{c}$ induced on the member.

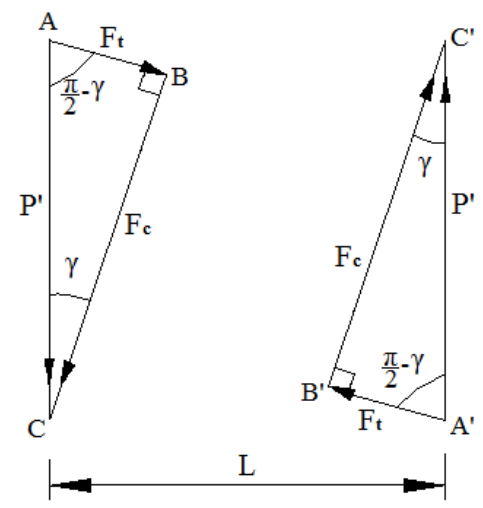

Figure $4 b$. Force triangle of $F_{t} \& F_{c}$ and their resultant $P$ ' equal and opposite to $P$.

From the force triangle shown in fig $4 \mathrm{~b}$, by the properties of triangles we can write,

$$
P^{\prime}=\sqrt{F_{t}^{2}+F_{c}^{2}}
$$

Since the horizontal component of the resultant will be zero as clearly shown in fig $4 \mathrm{~b}$

$P^{\prime}=F_{t} \sin \gamma+F_{0} \cos \gamma$

These equations can be further resolved as:

$$
\begin{aligned}
& P^{\prime}=\sqrt{\left(\frac{1-\cos \gamma}{\cos \gamma}\right)^{2} E^{2} A^{2}+(1-\cos \gamma)^{2} E^{2} A^{12}} \\
& P^{\prime}=\left(\frac{1-\cos \gamma}{\cos \gamma}\right) E \sqrt{A^{2}+\cos ^{2} \gamma A^{1^{2}}}
\end{aligned}
$$

And from eq (7b), we get,

$$
\begin{aligned}
P^{\prime} & =F_{t} \sin \gamma+F_{0} \cos \gamma \\
P^{\prime} & =\left(\frac{1-\cos \gamma}{\cos \gamma}\right) E A \sin \gamma+(1-\cos \gamma) E A^{\prime} \cos \gamma \\
& P^{\prime}=\left(\frac{1-\cos \gamma}{\cos \gamma}\right) E\left[A \sin \gamma+A^{s} \cos ^{2} \gamma\right]
\end{aligned}
$$

As we know that shear stress,

$$
\tau=\frac{P}{A}
$$

Now dividing equation (8) by A, we will get the value of $\tau$ as in eq (10).

$$
\begin{aligned}
\tau= & \frac{P}{A} \\
\tau= & \frac{1}{A}\left(\frac{1-\cos \gamma}{\cos \gamma}\right) E \sqrt{A^{2}+\cos ^{2} \gamma A^{\gamma^{2}}} \\
& \tau=\left(\frac{1-\cos \gamma}{\cos \gamma}\right) \cdot E \cdot \sqrt{1+\cos ^{2} \gamma\left(\frac{A}{A}\right)^{2}}
\end{aligned}
$$

By dividing equation (9) by A, we will get,

$$
\begin{aligned}
\tau= & \frac{P}{A} \\
\tau= & \frac{1}{A}\left(\frac{1-\cos \gamma}{\cos \gamma}\right) E\left[A \sin \gamma+A \cos ^{2} \gamma\right] \\
& \tau=\left(\frac{1-\cos \gamma}{\cos \gamma}\right) E\left[\sin \gamma+\frac{A^{\alpha}}{A} \cos ^{2} \gamma\right]
\end{aligned}
$$

So the equation (11) \& (12) shows a tremendous relation between the shear stress and the normal stress according to the classical shear model.

\section{SHEAR STRESS MODEL- YJ'S MODEL:}

We saw the classical model and realized that the shear stress was actually a combined result of normal stresses. But in this we are trying to show how this combined result of normal stresses actually [3]. Let us see how this model looks like: 


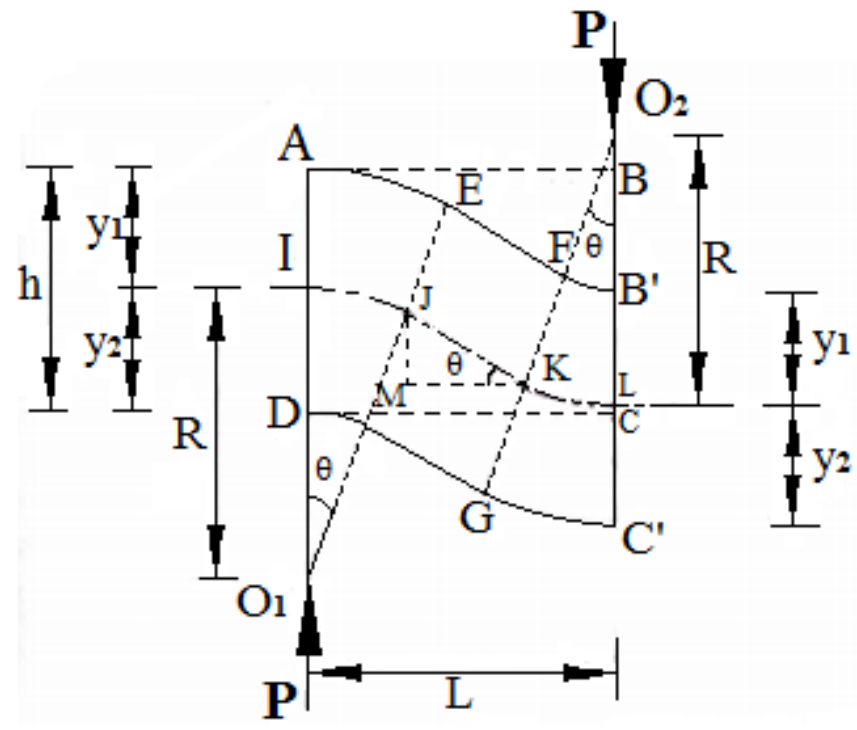

Figure 5- Representation o9f YJ's Shear Stress Model

In this model we think some bending also takes place at the ends. In figure 5, AEHD an FB'C'G represents that bending region. And in that bending $\mathrm{IJ}$ and $\mathrm{KL}$ represents the neutral axis and as we know that at neutral axis stress is zero in magnitude. In AEJI and KLC'G tensile stresses are distributed linearly. And in DHJI and FB'LK compressive stresses are distributed linearly, from zero at neutral axis to maximum at the end. Also,

$\theta \leftrightarrow$ Angle of bending and angle of deflection $=\angle A O_{1} \mathrm{E}=\angle C^{\prime} \mathrm{O}_{2} \mathrm{G}$

$R \leftrightarrow$ Radius of bending $=O_{1} \mathrm{I}=O_{1} \mathrm{~J}=O_{2} \mathrm{~L}=O_{2} \mathrm{~K}$

$L \leftrightarrow$ Horizontal length $=A B=C D$

$h \leftrightarrow$ Vertical length $=A D=B C=B^{\prime} C$

$y_{1} \leftrightarrow$ Vertical distance from NA to upper surface

$y_{2} \leftrightarrow$ Vertical distance from $N A$ to lower surface

$\mathrm{y}_{1}$ and $\mathrm{y}_{2}$ are not necessarily equal i.e. they may or may not be equal, it depends upon geometry such as triangular crosssection, T-shaped cross-section, etc. Now, every dimension which is shown in figure 6 are constant except $R$ and $\theta$ somehow depends upon the load $\mathrm{P}$ and some other properties of material like Young's modulus, area moment of inertia, etc. Now let us investigate the relation between $R$ $\& \theta$.

2.1 Radius and angle of Bending:

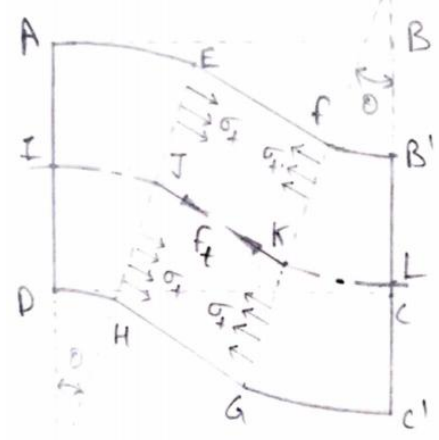

Figure 6a. Representation of tensile force \& tensile stress acting in region EFGH.

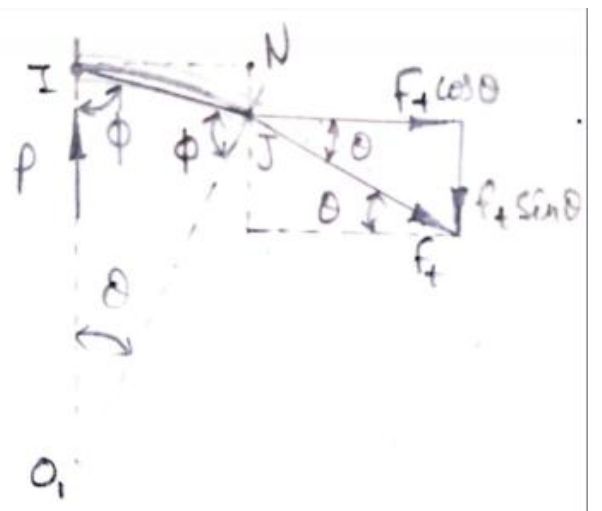

Figure $6 \mathrm{~b}$ Forces acting for bending region.

As we can see figure $6 \mathrm{~b}$ represents that is bending region two forces are acting viz. $P$ which is external load and $F_{t}$ which is internal tensile force. This is clear that this is like a cantilever beam [4]. The bending is also caused by the internal forces as well, so the moment acting at $\mathrm{J}$ would be internal moment,

$$
M_{1}=(\overline{I N}) \cdot F_{t} \cdot \sin \theta
$$

But, the total moment acting would be external moment, $M_{2}=P . l$

From the geometry, length of arc IJ is greater than length of line IJ, so in $\Delta O_{1} J I$, since $\quad \overline{I O_{1}}=\overline{j O_{1}}=R$

$\theta+\phi+\phi=\pi$

$\phi=\frac{\pi}{2}-\frac{\theta}{2}$

$\phi=\angle O_{1} I J=\angle O_{1} I I$, because $\Delta O_{1} I J$ is isosceles. 
By law of triangle,

$$
\begin{aligned}
& \frac{\overline{I J}}{\sin \theta}=\frac{R}{\sin \phi} \\
& \overline{I J}=\frac{R \sin \theta}{\sin \phi}=\frac{R \sin \theta}{\sin \left(\frac{\pi}{2}-\frac{\theta}{2}\right)}=\frac{R \sin \theta}{\cos \left(\frac{\theta}{2}\right)} \\
& \overline{I J}=\frac{R\left[2 \cdot \sin \left(\frac{\theta}{2}\right) \cos \left(\frac{\theta}{2}\right)\right]}{\cos \left(\frac{\theta}{2}\right)} \\
& \overline{I j}=2 \cdot R \cdot \sin \left(\frac{\theta}{2}\right)
\end{aligned}
$$

Since, arc

$\widehat{l j}=R_{*} \theta$

So from above equations,

$\widehat{\text { Ij }} \neq \overline{\text { Ij }}$

Now in $\Delta O_{1} l J$ and $\Delta I J N$

$\angle O_{1} I N=\frac{\pi}{2}$

$\angle H N=\frac{\pi}{2}-\phi=\frac{\pi}{2}-\frac{\pi}{2}+\frac{\theta}{2}=\frac{\theta}{2}$

Now,

$\overline{I N}=\bar{I} \cos (\angle J I N)$

$\overline{I N}=\left(2 R \cdot \sin \frac{\theta}{2}\right)\left(\cos \frac{\theta}{2}\right)$

$\overline{I N}=R \cdot \sin \theta$

Similarly,

$\overline{J N}=\overline{I J} \sin (\angle J I N)$

$$
\overline{7 N}=\left(2 R \cdot \sin \frac{\theta}{2}\right)\left(\sin \frac{\theta}{2}\right)
$$

$\overline{j N}=R \cdot(1-\cos \theta)$

So from equation (13a) \& (14c)

$M_{1}=(\overline{I N}) \cdot F_{t} \cdot \sin \theta$

$$
M_{1}=(R \cdot \sin \theta)\left(F_{t} \sin \theta\right)
$$

$M_{1}=F_{t} R \cdot \sin ^{2} \theta$

Now from equation (14a), $F_{t}$ is internal force as so we need to find $\sigma_{t}$ first, and

$F_{t}=\sigma_{\mathrm{t}} A$

Where, $A \leftrightarrow$ area $\perp$ to $\sigma_{t}$

$\sigma_{\mathrm{t}}=E \varepsilon_{\mathrm{t}}$

Where, $E \leftrightarrow$ Young s modulus

And, $\quad \epsilon_{\mathrm{t}} \leftrightarrow$ tensile strain at region EFGH

$\epsilon_{\mathrm{t}}=\frac{\overline{J K}-\overline{M K}}{\overline{M K}}=\frac{\overline{J K}-\overline{J K} \cos \theta}{\overline{J K} \cos \theta}$

$e_{\mathrm{t}}=\frac{1-\cos \theta}{\cos \theta}$

By combining equations (15b), (c) \& (d)

$F_{\mathrm{t}}=E A\left(\frac{1-\cos \theta}{\cos \theta}\right)$
From equation (15a)

$M_{1}=\operatorname{EAR}\left(\frac{1-\cos \theta}{\cos \theta}\right) \sin ^{2} \theta$

Now from the deflection equation,

EI $\frac{d^{2} y}{d x^{2}}=M_{1}$

EI $\frac{d y}{d x}=M_{1} x+C_{1}$

$E I y=\frac{M_{1} x^{2}}{2}+C_{1} x+C_{2}$

Applying boundary conditions i.e.

At, $x=0, y=0$

From equation (16c), $C_{2}=0$

At, $x=0, \frac{d y}{d x}=0$

From equation (16b), $C_{1}=0$

$\Rightarrow \quad$ Ely $=\frac{M_{1} x^{2}}{2}$

And here, $=\overline{I N}=R \sin \theta, y=\overline{J N}=R(1-\cos \theta)$

$\operatorname{EIR}(1-\cos \theta)=\frac{M_{1} R^{2} \sin ^{2} \theta}{2}$

$E I(1-\cos \theta)=\frac{M_{1} R \sin ^{2} \theta}{2}$

$E I(1-\cos \theta)=\left[\operatorname{EAR}\left(\frac{1-\cos \theta}{\cos \theta}\right) \sin ^{2} \theta\right]\left(\frac{R^{2} \sin ^{2} \theta}{2}\right)$

$\frac{2 I \cos \theta}{A}=R^{2} \sin ^{4} \theta$

$$
R^{2}=\frac{2 I \cos \theta}{A \sin \theta}
$$

Or,

$$
R=\frac{1}{\sin ^{2} \theta} \sqrt{2 I \frac{\cos \theta}{A}}
$$

This equation shows that $\mathrm{R}$ and $\theta$ are inter dependent variables. Let us find upon what they both depends:

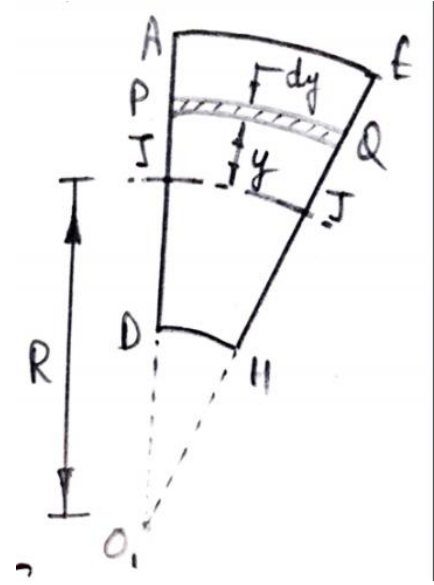

Figure 7- Bending region.

Published By:

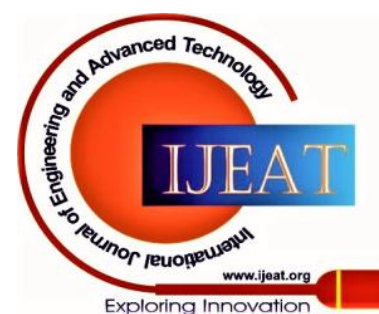




\section{Systematic Model Generation for Shear Stress using Elementary Mathematical Equation}

As shown in fig let us take an element PQ, which is $y$ distance from neutral axis and has dy thickness. So strain at PQ

$$
\begin{gathered}
\epsilon=\frac{\overline{P Q}-\overline{I j}}{\overline{I y}}=\frac{[(R+y) \theta-R \theta]}{R \theta} \\
\epsilon=\frac{y}{R}
\end{gathered}
$$

$$
\sigma=\frac{P l y}{I}
$$

So stress at $\mathrm{PQ}, \sigma=E l$

$$
\sigma=\frac{E y}{R}
$$

Also for bending stress can be determined by, $\sigma=\frac{M_{2} y}{I} \quad$ [Since, both moments $M_{1} \& M_{2}$ are acting and $M_{2}$ is internal]

So from equation $(17 \mathrm{c} \& \mathrm{~d})$,

$$
\frac{E y}{R}=\frac{P l y}{-}
$$

$$
R=\frac{E I}{P l}
$$

For $\theta$ combining all equations,

$\frac{\sin ^{4} \theta}{\cos \theta}=\frac{2}{A I}\left(\frac{P l}{E}\right)$

From equation (13)(b-),
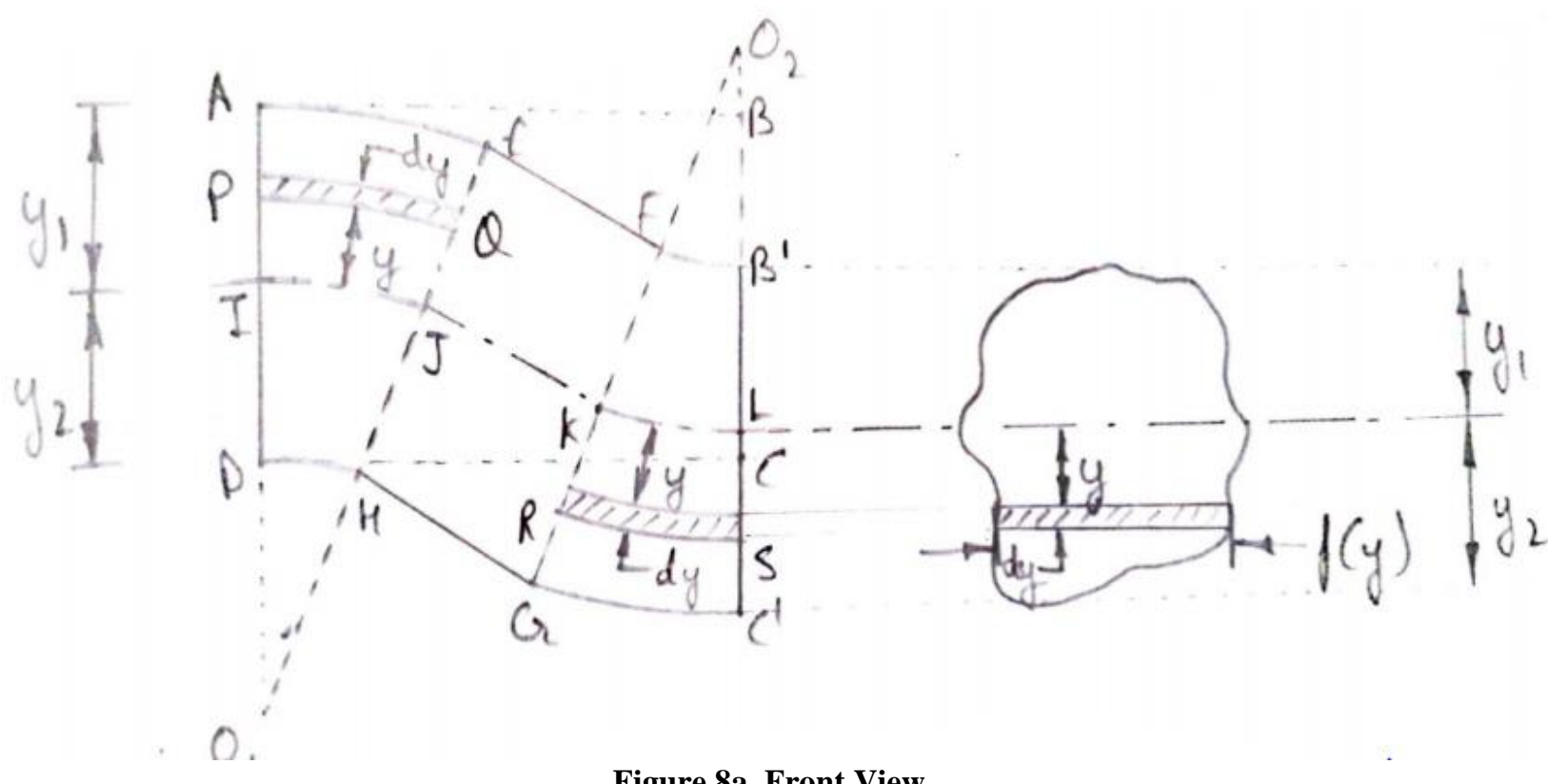

2.2 Forces due to Bending:

Figure 8a. Front View

Fig shows the front and side views, fig shows that the cross-section can be of any geometry and at $y$ distance from neutral axis, the width of the cross-section would be $f(y)$ which is the width function of that geometry [5]. It is different for different geometries and depends only upon the geometry, for example for rectangle $f(y)$ will be constant, for circle $f(y)=\sqrt{R^{2}-y^{2}}$, for triangle $f(y)=\left(\frac{2 h}{a}-y\right) \frac{b}{h}$ or $(y)=\left(\frac{2 h}{a}+y\right) \frac{b}{\hat{h}}$, etc.

Now, let $\sigma_{1}=$ stress at tension

$$
\begin{aligned}
& \sigma_{2}=\text { stress at compression } \\
& \sigma_{1}=\frac{P l y}{I} \\
& \text { And } \sigma_{2}=\frac{P l y}{I} \\
& \text { i.e. } d f_{1}=\sigma_{1} d A \\
& \& d f_{2}=\sigma_{2} d A
\end{aligned}
$$

Where, $\mathrm{df}_{1}=$ elementary force at area of $\mathrm{dA}$ (tensile)

$$
\mathrm{df}_{2}=\text { elementary force at area of } \mathrm{dA} \text { (compressive) }
$$
also,

$$
d A=f(y) d y
$$

for upper part, from eq 22, a, b \& c $F_{1 u}=\frac{P l}{I} \int_{0}^{y_{1}} f(y) \cdot y \cdot d y \quad \& \quad F_{2 u}=\frac{P l}{I} \int_{0}^{y_{1}} f(y) \cdot y \cdot d y$ And for lower part,

$F_{1 L}=\frac{P l}{I} \int_{0}^{y_{2}} f(y) \cdot y \cdot d y \quad \& \quad F_{2 L}=\frac{-P l}{I} \int_{0}^{y_{2}} f(y) \cdot y \cdot d y$ Now due to function $f(y) \&$ the limits $y_{1} \& y_{2}$, it's a fact that $F_{1 u}=F_{1 L} \& F_{2 u}=F_{2 L}$, so tensile force,

$$
F_{1}=\frac{P l}{I} \int_{0}^{\bar{y}} f(y) \cdot y \cdot d y
$$

And the compressive force,

$$
F_{2}=\frac{P l}{I} \int_{0}^{\bar{y}} f(y) \cdot y \cdot d y
$$

Where, $\bar{y} \leftrightarrow$ centroidal distance

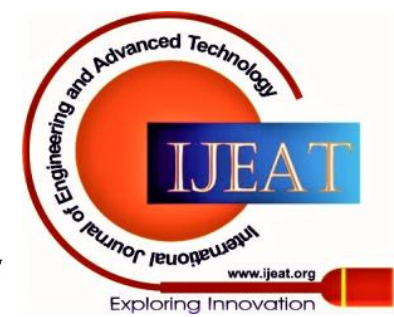




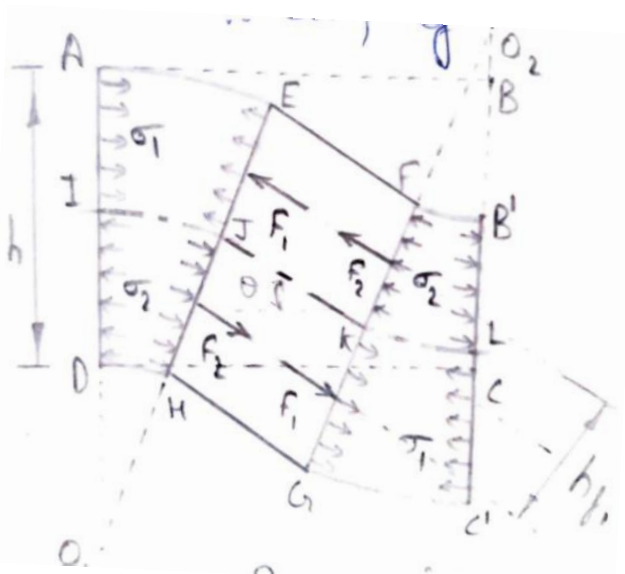

Figure 9a. Representation of forces \& stress.
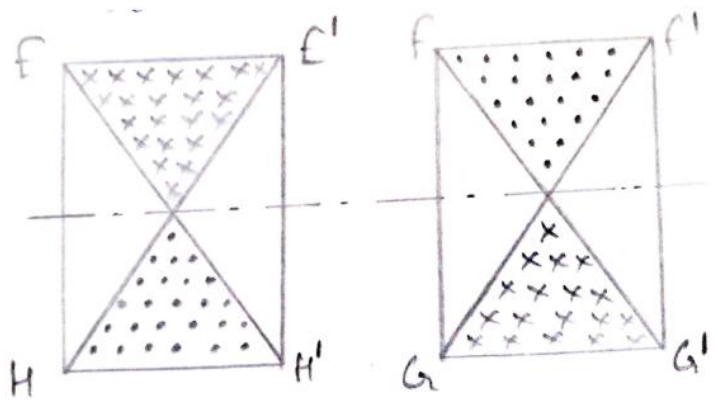

Figure 9b. Representation of stresses in side view w.r.t. neutral axis.

Now from the above figures we know the directions of each force which is $\theta$ from the horizontal. But we should also find out their positions as well. From figure $9 \mathrm{~b}$ we can see that the stress varies with the distance from the neutral axis i.e. it is zero at neutral axis and maximum at the ends. As we know that the stress equation for bending.

$$
\sigma=\frac{P l y}{I} \quad \text { or } \quad \sigma=k y
$$

Where,

$$
\begin{gathered}
k=\frac{P l}{I} \\
F=\int \sigma_{x} d A
\end{gathered}
$$

$\&$ force

$F=k \int y \cdot d A$

$F=k \int y \cdot f(y) \cdot d y$

So function $\left(\int y \cdot f(y) \cdot d y\right)$ gives the force distribution function. As we find centroid of any geometry by:

$\bar{y}=\frac{\sum A y}{\sum A}$

Here,

$A=\int y \cdot F(y) \cdot d y$

So the distance between the resultant of $F_{1} \& F_{2}$ and these twin forces would be $\mathrm{h}_{\mathrm{f} 1}$ distance apart.

$$
h_{f 1}=\frac{\bar{y}_{0}^{\bar{y}} y^{2} \cdot f(y) d y}{\int_{0}^{\bar{y}} y \cdot f(y) d y}
$$

And the resultant of $F_{1} \& F_{2}$ will be $F_{R 1}$ and from figure

$$
\begin{gathered}
F_{R 1}=F_{1}+F_{2} \\
F_{R I}=\frac{2 P I}{I} \int_{0}^{\bar{y}} y \cdot f(y) \cdot d y
\end{gathered}
$$

Now we got the magnitude, direction and the position of $F_{R I}$.

\subsection{Forces at region without Bending:}

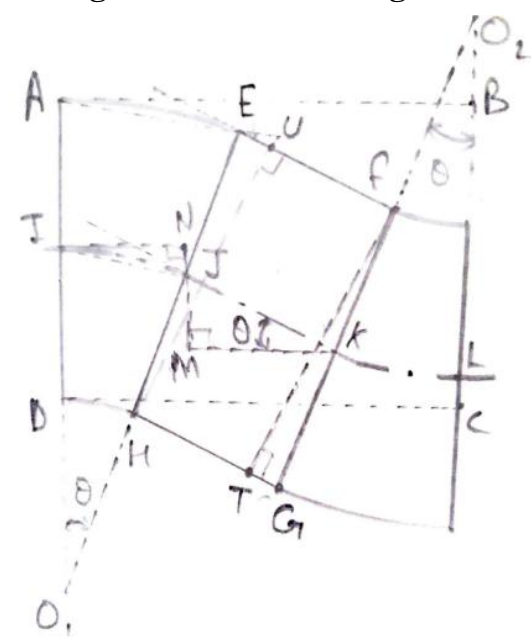

Figure 10a. Shear stress model with some perpendicular line segment FT, JM \& IN.

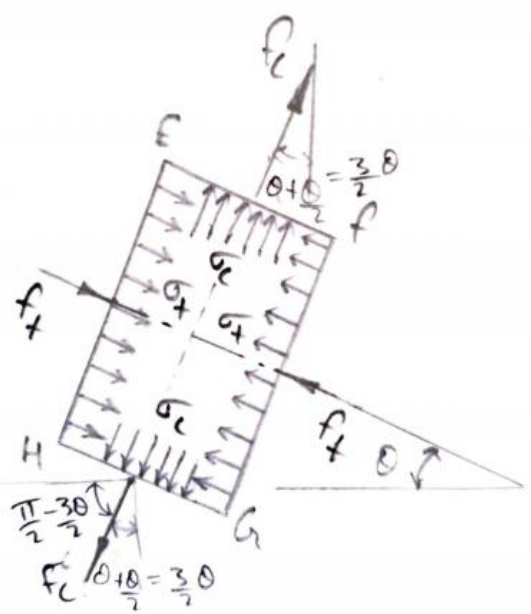

Figure 10b. Stress \& Forces acting on region EFGH.

As we saw in the classical shear stress model that there were also normal stresses involved, similarly in region EFGH tensile stress $\sigma_{t}$ and compressive stress $\sigma_{e}$ are involved. So just like equations $4 \mathrm{a} \& 4 \mathrm{~b}$ the respective tensile and compressive strains $\left(\epsilon_{t} \& \epsilon_{c}\right)$ can be determined as [6]:

Compressive strain,

$\epsilon_{c}=\frac{\overline{F G}-\overline{F I}}{\overline{F G}}$

For this first we need to find $\angle G F T$, from equation (17a)

$\angle O_{1} I J=\phi=\frac{\pi}{2}-\frac{\theta}{2}$

By further investigation we found that,

$\triangle I N]=\triangle E H U=\triangle G F T$

$\angle N I J=\angle G F T$

$\angle N I J+\angle O_{1} I J=\frac{\pi}{2}$

$\angle N I J+\frac{\pi}{2}-\frac{\theta}{2}=\frac{\pi}{2}$

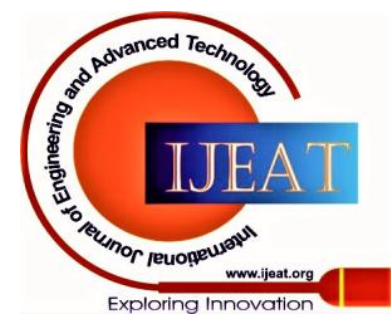




\section{Systematic Model Generation for Shear Stress using Elementary Mathematical Equation}

$\Rightarrow$

$$
\angle N I J=\frac{\theta}{2} \& \angle G F T=\frac{\theta}{2}
$$

$\epsilon_{c}=\frac{\overline{F G}-\overline{F G} \cos G G F T}{\overline{F G}}=\frac{\overline{F G}-\overline{F G} \cos \frac{\theta}{2}}{\overline{F G}}$

$\epsilon_{e}=1-\cos \frac{\theta}{2}$

Now Tensile strain,

$$
\begin{aligned}
& \epsilon_{\mathrm{t}}=\frac{\overline{\sqrt{K}}-\overline{M K}}{\overline{M K}}=\frac{\overline{\sqrt{K}}-\overline{\sqrt{N K}} \cos \theta}{\overline{\sqrt{K}} \cos \theta} \\
& \epsilon_{\mathrm{t}}=\frac{1-\cos \theta}{\cos \theta}
\end{aligned}
$$

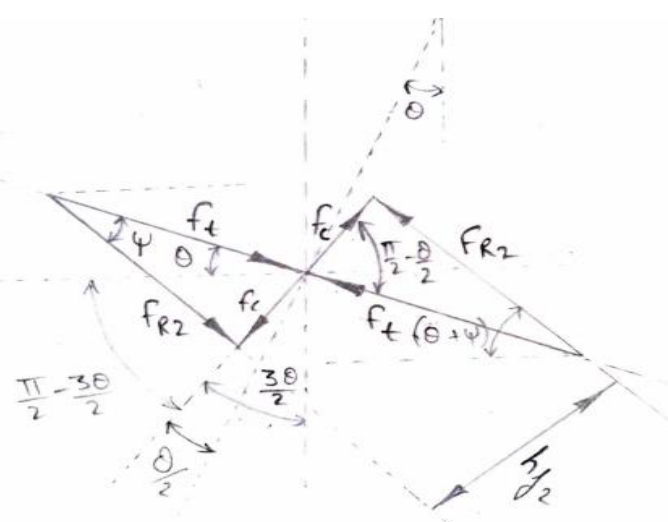

Figure 11b Detailed representation of $F_{t} \& F_{c}$ with their resultant $\mathbf{F}_{\mathrm{R} 2}$.

Where $\theta$ can be determined by equation (20b)

$$
\begin{aligned}
& \Rightarrow \\
& F_{\mathrm{t}}=E\left(\frac{1-\cos \theta}{\cos \theta}\right) \cdot \int_{0}^{\mathrm{h}} f(y) \cdot d y
\end{aligned}
$$

$$
\begin{aligned}
& \text { Here, } \\
F_{t}=E\left(\frac{1-\cos \theta}{\cos \theta}\right) & h_{f 2}=(l-2 R \theta) \cdot \sin \psi \\
& \text { Now the angle between } F_{t} \& F_{c} \text { is }\left(\frac{\pi}{2}-\frac{\theta}{2}\right) . \text { By applying } \\
& \text { parallelogram law, }
\end{aligned}
$$

$$
F_{R 2}=\sqrt{F_{t}^{2}+F_{c}^{2}-2 F_{t} \cdot F_{c} \cdot \cos \left(\frac{\pi}{2}-\frac{\theta}{2}\right)}
$$

$$
F_{c}=E\left(1-\cos \frac{\theta}{2}\right) A^{t}=\sqrt{F_{t}{ }^{2}+F_{c}^{2}-2 F_{t} \cdot F_{c} \cdot \sin \frac{\theta}{2}}
$$

$A^{v}=\left(\right.$ area $\left.\perp \sigma_{c}\right)=\overline{j K} \cdot f(\bar{y})$

$\left(\begin{array}{c}\mathrm{x}(\bar{y})=\text { width at centroidal distance } \\ \& \quad \overline{J K}=l-2 R \theta\end{array}\right)$

$$
\begin{gathered}
A^{y}=(l-2 R \theta) \cdot f(\bar{y}) \\
F_{c}=E\left(1-\cos \frac{\theta}{2}\right) \cdot(l-2 R \theta) \cdot f(\bar{y})
\end{gathered}
$$

So equation $(21 \mathrm{a} \& \mathrm{~b})$ gives the value of forces $F_{t} \& F_{c}$, now we need to find the direction and its position. From above equations in most cases $F_{c}$ would be rather smaller as compared to $F_{\mathrm{t}}$. Also $F_{\mathrm{t}}$ makes $\theta$ angle with horizontal and $F_{c}$ makes $\frac{\pi}{2}-\frac{a \theta}{2}$ with the horizontal.

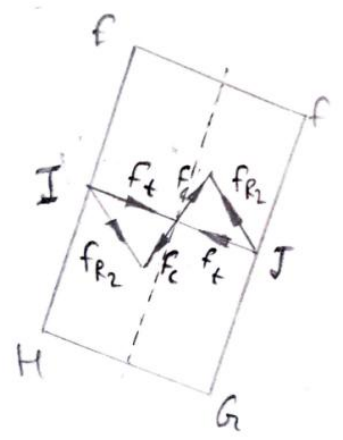

Figure 11a. Position of forces $F_{t} \& F_{c}$.
For $\psi$ which is the angle between $F_{R 1} \& F_{R 2}$,

$$
\begin{gathered}
\psi=\tan ^{-1}\left[\frac{F_{L} \sin \left(\frac{\pi}{2}-\frac{\theta}{2}\right)}{F_{L}-F_{L} \cos \left(\frac{\pi}{2}-\frac{\theta}{2}\right)}\right] \\
\left.\psi=\tan ^{-1}\left[\frac{F_{c} \cdot \cos \frac{\theta}{2}}{F_{t}-F_{c} \cdot \sin \frac{\theta}{2}}\right]\right]
\end{gathered}
$$

\subsection{Generated Force:}

Now from the previous equations we know the magnitude, direction and position of $F_{R_{1}} \& F_{R_{2}}$ The resultant of their force would be a generated force $F_{g}$ as shown below: 


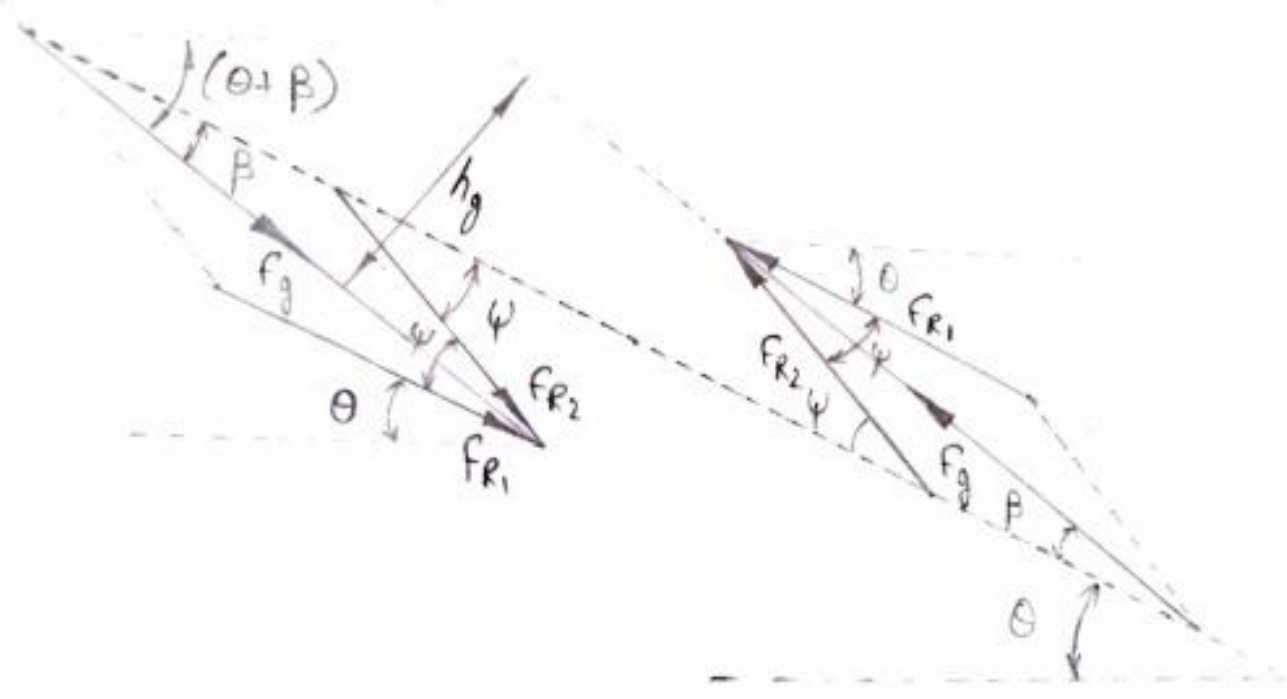

Figure 12-Representation of Generated force .

Since $F_{R_{1}}$ was already inclined at $\theta$ angle from horizontal and $F_{R_{2}}$ was inclined at $\theta+\psi$ angle, so the angle between $F_{R_{1}} \& F_{R_{2}}$ is $\psi$.

So the parallelogram law

$F_{g}=\sqrt{F_{R_{1}}{ }^{2}+F_{R_{2}}{ }^{2}+2 \cdot F_{R_{1}} \cdot F_{R_{2}} \cdot \cos \psi}$

(23a)

And the angle $\beta$ between $F_{R_{1}}$ and $F_{g}$ would be:

$\beta=\tan ^{-1}\left[\left(F_{R_{2} \times} \sin \psi\right) / F_{R_{1}}+F_{R_{2}} \times \cos \psi\right]$

(23b)

If $h_{g}$ is the perpendicular distance between $F_{g} \&$ its twin then,

$$
\begin{aligned}
& F_{R_{1}} \cdot h_{f_{1}}+F_{R_{2}} \cdot h_{f_{2}}=F_{g} \cdot h_{g} \\
& h_{g}=\left(F_{R_{1}} \cdot h_{f_{1}}+F_{R_{2}} \cdot h_{f_{2}}\right) / F_{g} \\
& \text { or } h_{g}=\frac{F_{R_{1}} \cdot h_{f_{1}}+F_{R_{2}} \cdot h_{f_{2}}}{\sqrt{F_{R_{1}}{ }^{2}+F_{R_{2}}{ }^{2}+2 \cdot F_{R_{1}} \cdot F_{R_{2}} \cdot \cos \psi}}
\end{aligned}
$$

Now the force $F_{g}$ is inclined at $(\theta+\beta)$ angle from

horizontal. Let $\theta+\beta=\omega$

Basically, $F_{g}$ is a totally internal force which is caused by external shear force 'P'. Similarly, $F_{g}$ will also cause some other internal force $F_{g}{ }^{t}$ at distance $h_{g}^{b}$ and inclined at $\omega^{t}$ angle from horizontal. And as we found $F_{g}, h_{g}$ and $\omega$ with respect to some quantities like $\mathrm{P}, \mathrm{L}, \mathrm{E}, \mathrm{I}, \mathrm{f}(\mathrm{y}), \theta$, etc. Similarly we can find $F_{g}^{v}, h_{g}^{v} \& \omega^{v}$. And $F_{g}{ }^{t}$ will then cause some other force $F_{g}{ }^{w}$ and then we will get:

$P=F_{g} \sin \omega+F_{g}^{t} \sin \omega^{g}+F_{g}^{s t} \sin \omega^{s t} \ldots \ldots \ldots \ldots$ or $\tau=\frac{1}{A}\left[F_{g} \sin \omega+F_{g}^{s} \sin \omega^{s}+F_{g}^{t s} \sin \omega^{t s}+F_{g}^{t s t} \sin \omega^{t s t} \ldots \ldots \ldots \ldots \ldots . . .\right.$.

All these equations can give us some important information about the stress distribution due to shear. The force acting or 'internal force' acting per unit area is what we call stress and could be found using those equations. We can also find the stress concentration in shear stress by this theory. As described in the last section that there is an infinite series of stresses, this is what I propose in the introduction part, to understand this let me give you an example of Taylor's expansion of any function in the introduction [7]. I think equation (25b) wouldn't look exactly like what I mentioned in the introduction, but it could give impressive results as I think.

The part where I mentioned in this page about 'stress concentration' [8] was about to calculate the distance between various generations of forces, magnitude of the forces and forces $F_{g}, F_{g}{ }^{\prime}, F_{g}{ }^{m}, F_{g}{ }^{m}$ etc. These are not single acting [9], these are the generations of forces and their

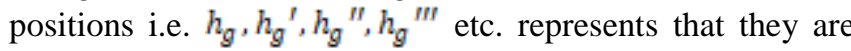
concentrated at that point i.e. the stress is distributed over that region above \& below it. It should be visualized clearly then only this theory could be understood properly [10].

\section{CONCLUSION}

The elaborative algebraic and derived equation for the shear stress is developed. The assumptions and the conditions used for the analysis and modeling are the general assumptions used normally. The efforts finally conclude that the developed model gives the equation similar to Taylor's expansion. All these equations can give us some important information about the stress distribution due to shear. This one equation finally leads to solve the large range of mathematical problems that falls under shear stress model. 
Further this model can be developed for uneven distribution of loads, force generations and complex geometry. The complete derivation is formulated using the understanding of classical geometry of the authors.

\section{ACKNOWLEDGEMENT}

The authors would like to thank Graphic Era Hill University, Graphic Era deemed to be University, all faculties and staff who helped and supported during the research activities.

\section{REFERENCES}

1. Natário, F., Ruiz, M. F., \& Muttoni, A. (2014). Shear strength of RC slabs under concentrated loads near clamped linear supports. Engineering structures, 76, 10-23.

2. Hirth, J. P., \& Cohen, M. (1970). On the strength-differential phenomenon in hardened steel. Metallurgical Transactions, 1(1), 3-8.

3. Qian, Y., \& Kawashima, S. (2018). Distinguishing dynamic and static yield stress of fresh cement mortars through thixotropy. Cement and Concrete Composites, 86, 288-296.

4. Ang, M. H., Wei, W., \& Teck-Seng, L. (1993, November). On the estimation of the large deflection of a cantilever beam. In Proceedings of IECON'93-19th Annual Conference of IEEE Industrial Electronics (pp. 1604-1609). IEEE.

5. Palermo, M., Pagliara, S., \& Bombardelli, F. A. (2020). Theoretical approach for shear-stress estimation at $2 \mathrm{D}$ equilibrium scour holes in granular material due to subvertical plunging jets. Journal of Hydraulic Engineering, 146(4), 04020009.

6. Bert, C. W. (1973). Simplified analysis of static shear factors for beams of nonhomogeneous cross section. Journal of Composite materials, 7(4), 525-529.

7. Nogueira, C. L., \& Rens, K. L. (2018). Experimental analysis of cement-based materials under shear stress. Construction and Building Materials, 170, 392-401.

8. De Leeuw, L. W., Diambra, A., Dietz, M. S., Mylonakis, G., \& Milewski, H. (2019). Interface shear strength of polypropylene pipeline coatings and granular materials at low stress level. In E3S Web of Conferences (Vol. 92, p. 13010). EDP Sciences.

9. Champagne, F. H., Harris, V. G., \& Corrsin, S. (1970). Experiments on nearly homogeneous turbulent shear flow. Journal of Fluid Mechanics, 41(1), 81-139.

10. Bynum, W. L. (1976). Weak parallelogram laws for Banach spaces. Canadian Mathematical Bulletin, 19(3), 269-275. 